\title{
Fragmentation of Positronium in Collision with He Atoms
}

\author{
S. Armitage, D. E. Leslie, A. J. Garner, and G. Laricchia* \\ Department of Physics and Astronomy, University College London, Gower Street, London, WCIE 6BT
}

(Received 29 June 2002; published 3 October 2002)

\begin{abstract}
The absolute cross section for the fragmentation of positronium in collision with $\mathrm{He}$ atoms has been measured. The results are compared with available theories. The longitudinal energy distributions of positrons resulting from fragmentation have also been determined and are found to display a peak situated just below half the residual energy. This is suggestive of the occurrence of "electron loss to the continuum" in which the two residual charged particles lie in a low relative-velocity Coulombcontinuum state.
\end{abstract}

DOI: $10.1103 /$ PhysRevLett.89.173402

PACS numbers: $36.10 . \mathrm{Dr}$

Positronium (Ps), the bound state of an electron $\left(e^{-}\right)$, and its antiparticle the positron $\left(e^{+}\right)$, is an interesting projectile: it is the lightest known atom (its mass being $\sim 10^{-3}$ that of conventional atoms), suggesting that recoil effects may be as important as in $e^{ \pm}$scattering [1]; it has no nucleus, the equal mass of its constituent particles resulting in the coincidence of its centers of charge and mass and, hence, in a zero static interaction with the undistorted target; its neutrality leads to zero firstorder polarization, as a consequence of which exchange effects play a comparatively bigger role in Ps than in $e^{-}$ scattering [2]. Depending on the spin orientation of its constituents, Ps may be formed in the ground state in an ortho- $\left({ }^{3} S_{1}\right)$ or para- $\left({ }^{1} S_{0}\right)$ state, the two being characterized by lifetimes differing by 3 orders of magnitude (142 ns and $125 \mathrm{ps,} \mathrm{respectively)} \mathrm{and} \mathrm{by} \mathrm{different} \mathrm{anni-}$ hilation modes (dominantly 3 - and $2-\gamma$, respectively).

Information on Ps collisions at room temperature may be obtained by studying its thermalization in a gas and measuring its lifetime [3], the Doppler shift of the energies of the annihilation quanta [4], or the deviation from collinearity between them [5,6]. At higher energies, cross sections can be measured directly using a Ps beam [7] produced by neutralizing a monoenergetic $e^{+}$beam in a gaseous target [8-10]. The beam produced in this manner consists essentially of ortho-Ps, the singlet state being virtually untransportable at atomic velocities due to its short lifetime. The angular and energy resolutions of the Ps beam depend on the neutralizing gas and, naturally, on the characteristics of the $e^{+}$beam itself [11]. To a first approximation (if no simultaneous inelastic effects occur [12] and if the ion recoil is negligible [13]), the kinetic energy of the Ps beam is expected to be given by $E=$ $E_{+}-\left(E_{i}-6.8 \mathrm{eV} / n^{2}\right)$ and is tunable via $E_{+}$, the positron incident energy, $E_{i}$ being the target ionization energy and $6.8 \mathrm{eV} / n^{2}$ the Ps binding energy in a state of principal quantum number $n$. At low energies, the decreasing positronium formation cross section and its increasing isotropy, together with the decreasing transport and detection efficiencies of Ps, have thus far prevented beam investigations below $\sim 10 \mathrm{eV}$. Until now, these investiga- tions have been restricted to total cross section measurements from simple target atoms and molecules, i.e., He, Ar, $\mathrm{O}_{2}$, and $\mathrm{H}_{2}[11,14,15]$. The determination of cross sections for Ps scattering are not just difficult for experimentalists, due to the very small projectile fluxes involved, but also for theorists, due to both the projectile and the target being composite objects. The problem is highlighted in the case of the Ps-He total cross section, where a discrepancy of a factor of 5 is found among the results of different theories [16-19] and different experiments $[4-6,14,15]$.

In this Letter, we present the results of the first direct experimental investigation of the fragmentation (or breakup) of Ps in collision with He atoms. Positrons emanating from Ps breakup have been detected and absolute Ps-He breakup cross sections have been determined. The results are compared with available theories. A measure of the longitudinal energy distributions of the resulting positrons has also been obtained. A peak in the distributions, observed at just below 50\% of the residual collision energy, suggests a strong postcollision interaction between the electron and the positron.

The experimental apparatus, shown schematically in Fig. 1, employs a commercially available radioisotope of sodium $\left({ }^{22} \mathrm{Na}\right)$ of activity 77.6. $\mathrm{mCi}$ as the source of $\beta^{+}$ particles. These are moderated using a rare-gas-solid (RGS) moderator [20] and accelerated to produce a tunable monoenergetic beam of positrons. The positrons are magnetically guided along the entire beam length using Helmoltz coils and separated from the flux of highenergy particles emanating from the source/moderator region by a Wien filter. The resulting monoenergetic $e^{+}$

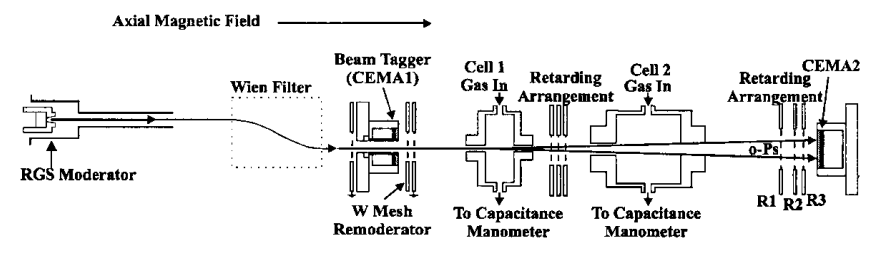

FIG. 1. Schematic diagram of the Ps beam. 
beam is fed into the experimental region, comprising a pair of gas cells and the detection system.

The $e^{+}$beam is converted into Ps via the chargeexchange reaction $e^{+}+A \rightarrow \mathrm{Ps}+A^{+}$with the target gas $(A)$ in the first gas cell. The neutralizing gas used for this study was $\mathrm{H}_{2}$ at a pressure of $10 \mu \mathrm{m} \mathrm{Hg}$ [15]. The retarding arrangement between the gas cells is used to prevent any positrons transmitted through the first gas cell from entering the second cell - the Ps scattering cell - containing the gas under investigation. Time-offlight (TOF) spectra [21] were obtained via coincidences between the signals of a "tagger" (CEMA1), incorporating a remoderator [22] and the end of beam detector (CEMA2). In front of CEMA2 are three retarding grids. The first (R1) may be used to bias off positrons (e.g., for total cross section measurements), the other two (R2 and R3) held at negative potentials, are used to increase the detection efficiency of CEMA2 by reflecting secondary $e^{-}$back towards the detector. As well as increasing the signal-to-background ratio, the TOF technique also enables the determination of the absolute incident energy of the Ps beam (to within $\pm 0.7 \mathrm{eV}$ ), its energy spread ( $\leq 5 \mathrm{eV}$ full width at half maximum) as well as monitoring its quantum state $(n=1$, under the present experimental conditions).

Positrons released from Ps breakup are confined to spiral trajectories in the guiding magnetic field with a Larmor radius given by their kinetic energy and emittance angle. If the Larmor radius is sufficiently smaller than the exit aperture of the scattering cell, the breakup positrons can exit and travel to the detector. Grounding the retarder R1 allows these positrons to reach the detector and be counted together with the transmitted Ps beam. The breakup $e^{+}$flux is obtained by taking two measurements: with R1 positive, only Ps is detected; with R1 grounded, both positrons and Ps are recorded in the spectrum. Subtraction of the two spectra (normalized for measuring time, gas pressure, and number of incident positrons) yields the flux of positrons $\left(N_{+}\right)$from the collisional breakup of Ps. No such signal was observed with vacuum in cell 2. Single-collision conditions were verified by checking the linear dependence of $N_{+}$with the pressure of He in cell 2.

In order to ensure that all positrons from Ps breakup reach the detector, the magnitude of the magnetic field $(B)$ in the scattering region was adjusted such that the Larmor radius of the breakup positrons plus the radius of the Ps beam was smaller than the radius of the exit aperture of the scattering cell. This was checked by verifying signal saturation with increasing values of $B$ and performing measurements at this saturation $B$ value.

The absolute breakup cross section has been obtained as follows:

$$
\sigma_{\text {bu }}(E)=\frac{N_{+}}{\left(N_{\mathrm{Ps}}\right)_{\mathrm{scatt}}} \sigma_{T}(E) S G\left(\frac{\varepsilon_{\mathrm{Ps}}}{\varepsilon_{+}}\right) .
$$

$\left(N_{\text {Ps }}\right)_{\text {scatt }}$ is the scattered Ps flux given by the difference between the measured incident- and attenuated-Ps fluxes, $\sigma_{T}$ is the total cross section of Ps of incident energy $E$, and $S$ accounts for inflight annihilation and corresponds to the ratio between the number of Ps atoms that traverse the scattering cell and the equivalent number that reach the detector. As Ps is neutral and thus unconfined by the magnetic field of the apparatus, the dimensions of the scattering cell limit the solid angle for its detection. However, Ps atoms that enter the scattering cell outside of the detection solid angle may break up, giving rise to positrons which may be magnetically confined and detected at CEMA2, leading to a greater breakup $e^{+}$flux than from the detectable Ps alone. The geometric factor $G$ in Eq. (1) allows for this effect. $G$ corresponds to the ratio of the average Ps solid angle through the scattering cell to the Ps solid angle defined by the entrance aperture of the scattering cell. $G$ has been determined to be 0.51 for our geometry. At 18 and $33 \mathrm{eV}$, measurements have been repeated with another gas cell designed such that $G=1$ and found to be in good agreement with the results obtained with the original cell.

The respective detection efficiencies of CEMA2 for Ps and the breakup positrons [ $\varepsilon_{\mathrm{Ps}}$ and $\varepsilon_{+}$, respectively, in Eq. (1)] have been determined by performing coincidence measurements between CEMA2 and a CsI $\gamma$-ray detector. In the case of Ps, two methods have been used. In the first, under the assumption that the dominant factor in determining the detection efficiency is the impact velocity, positrons of the same velocity as the Ps beam were used. In the second, Ps was used directly. The two methods, which will be described in detail elsewhere [23], yield results that are, within errors, in agreement with each other. The values of $N_{+} /\left(N_{\mathrm{Ps}}\right)_{\text {scatt }}, \sigma_{T}$, and $S$ used in the determination of the absolute breakup cross section are shown in Table I. In this table, the errors represent the uncertainty on the weighted mean of different measurements. They comprise statistical errors arising from particle counting techniques and pressure $( \pm 2 \%)$. The values for $\sigma_{T}$ shown in Table I were determined simultaneously in this work and found to be in good agreement with previous beam measurements $[14,15]$.

Figure 2 shows the absolute breakup cross sections for Ps-He scattering obtained using the equivelocity $e^{+}$

TABLE I. Values for the quantities used in the determination of the absolute breakup cross section as in Eq. (1). The flight lengths were 0.393 and $0.293 \mathrm{~m}$ at the two highest and lowest energies, respectively.

\begin{tabular}{ccccc}
\hline \hline Energy $(\mathrm{eV})$ & $N_{+} /\left(N_{\mathrm{Ps}}\right)_{\text {scatt }}$ & $\sigma_{T}\left(10^{-20} \mathrm{~m}^{2}\right)$ & $\varepsilon_{\mathrm{Ps}} / \varepsilon_{+}$ & $S$ \\
\hline 33 & $4.5 \pm 0.9$ & $3.2 \pm 0.3$ & $0.30 \pm 0.01$ & 0.455 \\
27 & $4.4 \pm 0.3$ & $5.0 \pm 0.3$ & $0.31 \pm 0.01$ & 0.404 \\
18 & $4.9 \pm 0.4$ & $4.7 \pm 0.4$ & $0.32 \pm 0.01$ & 0.506 \\
13 & $9.2 \pm 1.2$ & $2.9 \pm 0.3$ & $0.33 \pm 0.01$ & 0.436 \\
\hline \hline
\end{tabular}


detection efficiencies, which have been chosen due to their greater statistical precision and the availability of experimental data for quantities needed in their evaluation [23]. The errors on the quantities in Table I were combined in the standard manner to obtain the error bars on the breakup cross sections as shown in Fig. 2. An additional uncertainty of $(\underset{-(20-30)}{+8}) \%$ is ascribed to the absolute values of the breakup cross section, due to the systematic uncertainties in the determination of the detection efficiencies [23]. A Ps beam flux of typically $10^{-2} \mathrm{~s}^{-1}$ constrained measurements in this initial study to only four incident energies.

In Fig. 2, the experimental determinations are compared with available theories. The theory of Biswas and Adhikari [17], which uses the Born approximation, is found to exceed experimental data by approximately a factor of 2, raising doubts on the level of the agreement previously found between their total cross sections and those from beam measurements [15].

Good agreement is found with the coupled-state theoretical determination of Blackwood et al. [16]. However, in the case of the total cross section, a significant discrepancy exists between this theory and the beam data, with theory lying below measurements by up to $30 \%$ above $10 \mathrm{eV}$. This may indicate either an underestimate of the elastic cross section and/or of target inelastic effects in Ps-He scattering. Recent work on Ps-H, He scattering [19,24-27] has indeed highlighted the importance of including (virtual and real) target inelastic channels in Ps scattering theory.

From the time-of-flight spectra of the positrons from Ps breakup, their longitudinal energy distribution has

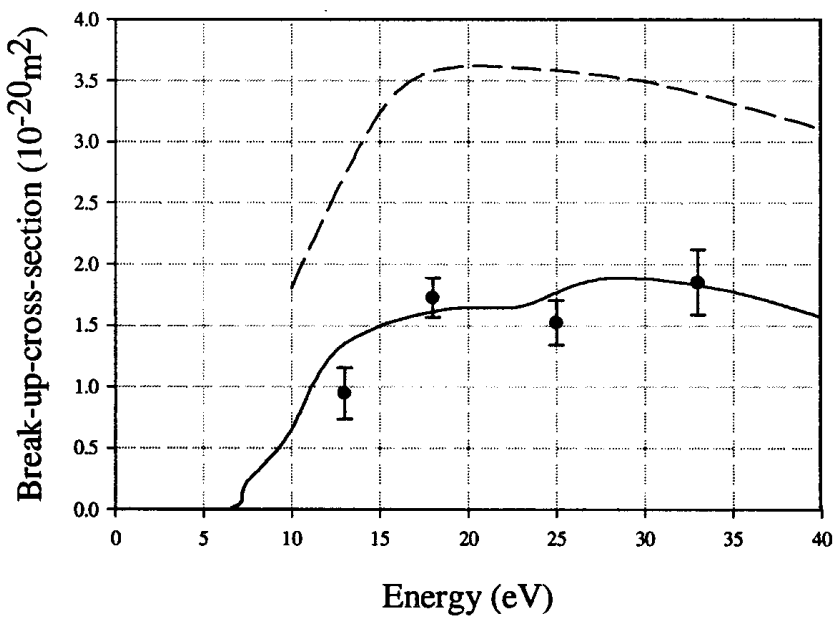

FIG. 2. Absolute breakup cross section for Ps-He scattering. Solid curve, Blackwood et al. [14]; dashed curve, Biswas and Adhikari [15]; solid circles, this work. An uncertainty of $\left(\begin{array}{c}+8 \\ -(20-30)\end{array}\right) \%$, additional to the error bars shown, is ascribed to the cross section due to uncertainties in the detection efficiencies (see text). also been obtained. Figure 3 shows the distributions corresponding to the four incident Ps energies investigated.

The data have been summed into $1 \mathrm{eV}$ bins and normalized to unity for shape comparison. A peak just below $50 \%$ of the residual energy $\left(E_{r}=E-6.8 \mathrm{eV}\right)$ becomes increasingly apparent in the spectra with increasing energy. The peak is suggestive of both final state particles (i.e., $e^{+}$and $e^{-}$) traveling in the forward direction with the same velocity, signaling the occurrence of electron loss to the continuum (ELC) [28], a phenomenon familiar in atom/ion collision physics [29]. The related process of electron capture to the continuum was first predicted for $e^{+}$impact by Brauner and Briggs $[30,31]$ and has recently been observed in $e^{+}-\mathrm{H}_{2}$ collisions [32,33]. Unlike ions which remain essentially undeflected by the collision and for which the phenomenon is manifested by a sharp cusp in the electron spectrum around $0^{\circ}$, positron impact gives rise to a small broad peak in the triply differential spectrum of the ejected electrons. This contrast corresponds to a mass effect due to the light scattered projectiles (and captured electrons) being distributed over a considerable angular range.

As the positrons released through Ps breakup are confined by the axial magnetic field, any finite angular distribution will result in a shift to lower values of the measured energy distributions and a corresponding broadening. The shift in the peak position from $E_{r} / 2$ suggests therefore that the breakup positrons are released within a small angle ( $\leq 20^{\circ}$ at the higher energies) with respect to the beam axis. Furthermore, the measured energy width cannot be entirely attributed to the system energy resolution $(\Delta E / E \sim 20 \%)$ and the incident Ps
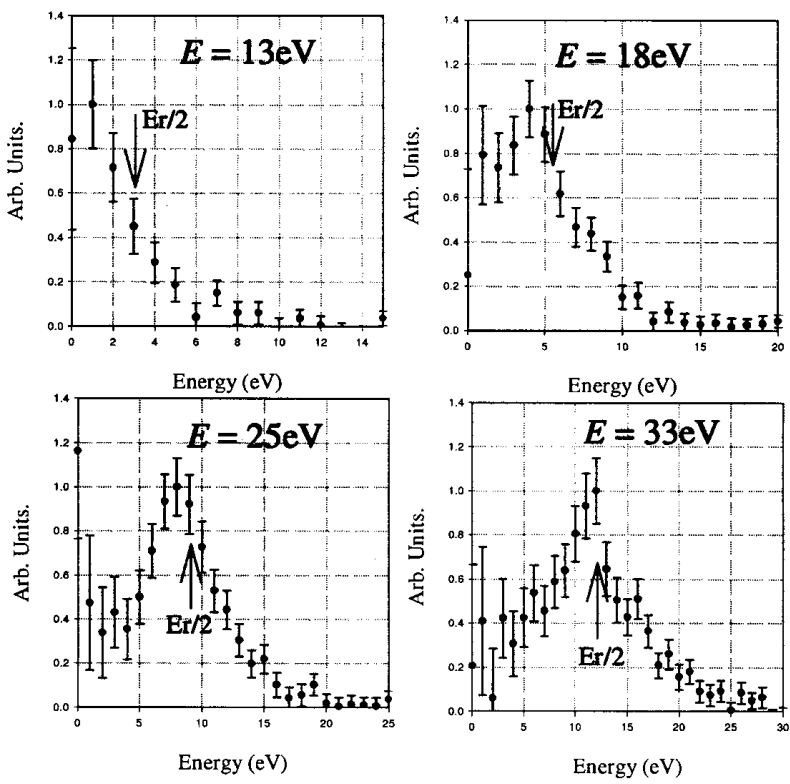

FIG. 3. Longitudinal energy spreads of the positrons released from Ps breakup. 
energy spread and thus also implies some angular/energy spread.

In conclusion, the absolute breakup cross section of Ps atoms in collision with $\mathrm{He}$ atoms has been measured for the first time and found to be in good agreement with a coupled-state calculation [16]. The longitudinal energy distributions of the final state positrons have also been measured and found to exhibit a peak a little below $E_{r} / 2$ suggesting the occurrence of electron loss to the continuum. Unlike the case of matter atoms, the phenomenon appears to occur over a significant angular range. Further work is required to decouple the energy distribution from the angular spread of the positrons released through Ps breakup. Future plans also aim to probe target inelastic (and doubly inelastic) events by monitoring electrons, as well as positrons, in the final state.

The Engineering and Physical Research Council is gratefully acknowledged for supporting this work under Grant No. GR/L96837 and for providing S. A. and D. E. L. with research support.

*Corresponding author.

Email address: g.laricchia@ucl.ac.uk

[1] J. R. Manson and R. H. Richie, Phys. Rev. Lett. 54, 785 (1985).

[2] For example, J. E. Blackwood, M.T. McAlinden, and H. R. J. Walters, Phys. Rev. A 65, 032517 (2002).

[3] For example, M. Charlton, Rep. Prog. Phys. 48, 737 (1985).

[4] M. Skalsey, J. J. Engbrecht, R. K. Bithell, R. S. Vallery, and D.W. Gidley, Phys. Rev. Lett. 80, 3727 (1998).

[5] P. G. Coleman, S. Rayner, F. M. Jacobsen, M. Charlton, and R. N. West, J. Phys. B 27, 981 (1994).

[6] Y. Nagashima, T. Hyodo, K. Fujiwara, and A. Ichimura, J. Phys. B 31, 329 (1998).

[7] N. Zafar, G. Laricchia, M. Charlton, and A. Garner, Phys. Rev. Lett. 76, 1595 (1996).

[8] B. L. Brown, Bull. Am. Phys. Soc. 30, 614 (1985); Positron(-electron)-Gas Scattering, edited by W. E. Kauppila, T.S. Stein, and J. M. Wadehra (World Scientific, Singapore, 1986), p. 212.

[9] G. Laricchia, M. Charlton, S. A. Davies, C. D. Beling, and T. C. Griffith, J. Phys. B 20, L99 (1987).
[10] G. Laricchia and N. Zafar, Solid State Phenom. 28 \& 29, 347 (1992).

[11] A. J. Garner, G. Laricchia, and A. Özen, Nucl. Instrum. Methods Phys. Res., Sect. B 143, 155 (1998).

[12] G. Laricchia and J. Moxom, Phys. Lett. A 174, 255 (1993).

[13] This may not always be a valid assumption; see, e.g., J. Fiol and R. E. Olson, J. Phys. B 35, 1173 (2002).

[14] A. J. Garner, A. Ozen, and G. Laricchia, J. Phys. B 33, 1149 (2000).

[15] A. J. Garner, G. Laricchia, and A. Özen, J. Phys. B 29, 5961 (1996).

[16] J. E. Blackwood, C. P. Campbell, M.T. McAlinden, and H. R. J. Walters, Phys. Rev. A 60, 4454 (1999).

[17] P. K. Biswas and S. K. Adhikari, Phys. Rev. A 59, 363 (1999).

[18] N. K. Sarker and A. S. Ghosh, J. Phys. B 32, 1657 (1999).

[19] A. Basu, P. K. Sinha, and A. S. Ghosh, Phys. Rev. A 63, 052503 (2001).

[20] A. Özen, A. J. Garner, and G. Laricchia, Nucl. Instrum. Methods Phys. Res., Sec. B 171, 172 (2000).

[21] N. Zafar, G. Laricchia, M. Charlton, and Y.C. Griffith, J. Phys. B 24, 4461 (1991).

[22] G. Laricchia, S. A. Davies, M. Charlton, and T.C. Griffith, J. Phys. E 21, 886 (1988).

[23] S. Armitage, D. E. Leslie, and G. Laricchia, Meas. Sci. Technol.(unpublished).

[24] J. E. Blackwood, M. T. McAlinden, and H. R. J. Walters, Phys. Rev. A 65, 032517 (2002).

[25] A. Basu, P. K. Sinha, and A.S. Ghosh, Phys. Rev. A 63, 012502 (2000).

[26] H. Ray and A. S. Ghosh, J. Phys. 31, 4427 (1998).

[27] C. P. Campbell, M. T. McAlinden, F. G. R. S. MacDonald, and H. R. J. Walters, Phys. Rev. Lett. 80, 5097 (1998).

[28] J. Ludlow and H. R. J. Walters, Many-Particle Spectroscopy of Atoms, Molecules, Clusters, and Surfaces, edited by Jo Berakdar and J. Kirschner (Kluwer, New York, 2001), p. 319.

[29] G. B. Crooks and M. E. Rudd, Phys. Rev. Lett. 25, 1599 (1970).

[30] M. Brauner, J. S. Briggs, and H. Klar, J. Phys. B 22, 2265 (1989).

[31] M. Brauner and J. S. Briggs, J. Phys. B 24, 2227 (1991).

[32] À. Kövér and G. Laricchia, Phys. Rev. Lett. 80, 5309 (1998).

[33] À. Kövér, K. Paludan, and G. Laricchia, J. Phys. B 34, L219 (2001). 